\title{
LOW PERIODICITY LATTICE FOR THIRD GENERATION LIGHT SOURCES: AVOIDANCE OF DYNAMIC APERTURE REDUCTION BY SEXTUPOLE COMPENSATION
}

\author{
Yu. Senichev*, ISA, Aarhus University, Denmark
}

\section{INTRODUCTION}

The classic ring structure of a number of identical achromat cells arranged in a highly symmetric circular form has been used in the vast majority of synchrotron light sources. However, lattices with small electron emittances require smaller dispersion function and stronger sextupoles are needed in order to correct the chromaticity. In the same time, as is well known, the sextupoles dramatically decrease the dynamic aperture due to their nonlinear action. To avoid the influence of sextupoles on the dynamic aperture the multi-family sextupole schemes are used. But, unfortunately, all of them do not give complete compensation. In particular, for third generation synchrotron light sources the residual sextupole component becomes the stronger factor in comparison with errors and misalignments and this factor limits the dynamic aperture. Thus, the search of new solutions to decrease the influence of the sextupoles has remained to be actual. But let us ask ourselves, if we can not suppress effectively the influence of sextupoles, may be we should properly use the non-linearity of sextupole to stabilize motion? Such an instrument can be the non-linear tune shifts. Obviously, to realize this idea we should step aside from the classic ring structure, since it does not permit us to control the sign and the magnitude of tune shift independently on the tunes themselves. We propose a lattice consisting of many arcs containing combined function (or usual) magnets and focusing (and defocusing) quadrupoles separated by identical optical channels consisting of either one, two or more dispersionless straight sections[1]. A significant advantage of such a design is the ability to separate the functions of the arcs and the straight sections. The periodical part of the arcs is a pseudo-second order achromat joined with the straight sections through a dispersion suppressor and it differs from the second order achromat[2] by non-zero chromaticity. The lattice has one focusing and one defocusing family of sextupoles located on the periodical part of the arc. Varying the chromaticity of arcs by sextupoles and the chromaticity of straight sections by quadrupoles and keeping the total chromaticity equal zero, we can modify the tune shift at any working point.

\section{SEXTUPOLE RESONANCES WITH NONLINEAR TUNE SHIFTS}

In the proposed lattice the highest multipole is a sextupole. The resonance arises under the condition $q+k_{x} \nu_{x}+k_{y} \nu_{y}=$

\footnotetext{
*Email: senichev@dfi.aau.dk
}

$\Delta \cdot\left(k_{x}^{2}+k_{y}^{2}\right)^{1 / 2}$. Already in the first order of the resonance theory $N=1$ the sextupole excites four resonances $\left\{k_{x}, k_{y}\right\}=\{1,0 ; 3,0 ; 1, \pm 2\}$. The number of resonances and their order grow with the the order $N$ as $2^{N+1}$. In action-angle variables the averaging Hamiltonian of the motion can be written as:

$$
\begin{aligned}
& H\left(I_{x}, I_{y}, \varphi_{x}, \varphi_{y}\right)= \\
& \frac{\left(k_{x}^{2}+k_{y}^{2}\right)^{1 / 2}}{k_{x}} \cdot \Delta \cdot I_{x}+\frac{\left(k_{x}^{2}+k_{y}^{2}\right)^{1 / 2}}{k_{y}} \cdot \Delta \cdot I_{y}+ \\
& 2\left\langle h_{k_{x}, k_{y}, q}\right\rangle I_{x}^{k_{x} / 2} I_{y}^{k_{y} / 2} \cos \left(k_{x} \varphi_{x}+k_{y} \varphi_{y}\right) \\
& +\zeta_{x} I_{x}^{2}+\zeta_{x y} I_{x} I_{y}+\zeta_{y} I_{y}^{2},
\end{aligned}
$$

where the Fourier harmonic $h_{k_{x}, k_{y}, q}$ determines the effective strength of the resonance $k_{x} \nu_{x}+k_{y} \nu_{y}=q$ under the detuning $\Delta$. It is proportional to the integral

$$
\left\langle h_{k_{x}, k_{y}, q}\right\rangle \sim \int_{s_{0}}^{s} \beta_{x}^{k_{x} / 2} \beta_{y}^{k_{y} / 2} K_{s}(s) e^{i\left(k_{x} \mu_{x}+k_{y} \mu_{y}\right)} d s,
$$

where $\mu_{x, y}=\int_{s_{0}}^{s} \frac{d s}{\beta_{x, y}}$ is the phase advance. Several formalisms of the perturbation theory up to the second order $[3,4]$ have been used to derive the expression of the tune shifts $\zeta_{x}, \zeta_{x y}, \zeta_{y}$ of the sextupoles. They all are the functions of the effective strength of the resonance and the detuning: $\zeta_{x, x y, y}=F\left(\frac{\left|h_{k_{x}, k_{y}, q}\right|^{2}}{k_{x} \nu_{x}+k_{y} \nu_{y}-q}\right)$. Thus, when we compensate the influence of the sextupoles, the strength of the resonance is suppressed proportionally to the first power of $h_{k_{x}, k_{y}, q}$, while the tune shifts are decreased as $\left|h_{k_{x}, k_{y}, q}\right|^{2}$.

\subsection{Lattice classification}

Let us consider the case of a third integer resonance in one plane. In the Hamiltonian system, where the friction force is absent, there are two types of fixed points. The fixed point $\overline{I_{x}}, \overline{\varphi_{x}}$ is the saddle, if the roots of the characteristic equation are real and it is the centre, when the roots are imaginary. The point itself is derived from the equations

$$
\begin{aligned}
\Delta+\frac{3}{2} h_{30 q} \overline{I_{x}^{1 / 2}} \cos 3 \overline{\varphi_{x}}+2 \zeta_{x} \overline{I_{x}}+\zeta_{x, y} I_{y} & =0 \\
\sin 3 \overline{\varphi_{x}} & =0
\end{aligned}
$$

The last gives us plenty of combinations with the fixed points depending on the ratio between the parameters $\Delta$, 
$h_{30 q}, \zeta_{x}$ and $\zeta_{x, y}$. The influence of the non-linearity is specified by the discriminant $D$ in the expression:

$\overline{I_{x}^{1 / 2}}=-\frac{3 h_{30 q} \cos 3 \overline{\varphi_{x}}}{8 \zeta_{x}} \pm \frac{1}{4 \zeta_{x}} \sqrt{\frac{9}{4} h_{30 q}-8 \zeta_{x}\left(\Delta+\zeta_{x, y} I_{y}\right)}$

Substituting $\overline{I_{x}}, \overline{\varphi_{x}}$ in the characteristic equation, we define what kind of fixed point it is.

From the lattice design point of view there are three interesting cases: $\zeta_{x} \ll h_{30 q} ; \zeta_{x} \sim h_{30 q}$ and $\zeta_{x} \gg h_{30 q}$.

The lattices with the small non-linearity $\zeta_{x} \ll h_{30 q}$ are classified among the first or second generation synchrotron light sources. The system has one centre and three saddles. Since the non-linearity is negligibly small, the dynamic aperture is determined by the value of $\overline{I_{x}} \leq\left(\frac{2 \Delta}{3 h_{30 q}}\right)^{2}$, when the motion is still stable. Under the resonance condition $\Delta=0$ the centre is degenerated into the saddle and the stable area shrinks into zero. In such a lattice the working point is kept as far from the resonance as possible. Usually these lattices have a small value of $h_{30 q}$ because the sextupoles are very weak and there is no problem with the dynamic aperture.

The lattices with $\zeta_{x} \sim h_{30 q}$ have the moderate nonlinearity. In common case the system has four islands with four centres and three saddles. Such a lattice is used for the second and third synchrotron light sources, where the sextupoles have much stronger gradient in comparison with previous generation. To compensate sextupoles the multifamilies sextupoles schemes are used. Since the tune shifts are proportional to $\left|h_{k_{x}, k_{y}, q}\right|^{2}$ and the working points are chosen far away from integer tune, the non-linearity are decreased even faster than the third harmonic. Really the dynamic aperture is determined by how successful we have been in simultaneous adjustment of the appropriate value of $\zeta_{x}$ and $h_{30 q}$.

The lattices with $\zeta_{x} \gg h_{30 q}$ have to be classified as a special lattice, since it is a case, when the value of $h_{30 q}$ is effectively suppressed, but the non-linearity remain to be under control and strong. It is obvious from (4), if the sign of the detuning $\Delta$ coincides with the sign of the tune shift $\zeta_{x}$, the discriminant is negative and the system has only one centre at $I_{x}=0$. Therefore this case corresponds to the maximum stable region and the lattice with these features is the most hopeful for the third and fourth generation source.

\subsection{Nekhoroshev's criterium}

So, in order to get the maximum stable region the sign of the non-linearity $\zeta_{x}$ has to be the same as the detuning $\Delta$. However, we can see from (4), if $\zeta_{x y}$ has the opposite sign with the tune $\Delta$, then under some amplitude of oscillation in the vertical plane $I_{y}$ the total detuning $\Delta_{\text {total }}=\Delta+\zeta_{x, y} I_{y}$ can make the discriminant $D \geq 0$. It means that the two dimensional vector remains to be on the resonant surface. In other words the non-linear system is unable to leave the res- onance at all and it behaves as quasi-isochronous system. The phenomena of quasi-isochronism for the nonlinear resonances was investigated by Nekhoroshev [5]. Let us apply Nekhoroshev' criterium to our two dimensional system. The non-resonant part of the Hamiltonian is $\delta H\left(I_{x}, I_{y}\right)=\zeta_{x} I_{x}^{2}+\zeta_{x y} I_{x} I_{y}+\zeta_{y} I_{y}^{2}$. The vector of frequencies passing through the point of the resonant surface $I^{r}=\left(I_{x}^{r}, I_{y}^{r}\right)$ is $\omega=\left(2 \zeta_{x} I_{x}+\zeta_{x y} I_{y} ; 2 \zeta_{y} I_{y}+\zeta_{x y} I_{y}\right)$. The quasi-isochronism condition by Nekhoroshev is fulfilled, when $k_{x}\left(2 \zeta_{x} I_{x}^{r}+\zeta_{x y} I_{y}^{r}\right)+k_{y}\left(2 \zeta_{y} I_{y}^{r}+\zeta_{x y} I_{y}^{r}\right)=$ 0 and $\zeta_{x} k_{x}^{2}+\zeta_{x y} k_{x} k_{y}+\zeta_{y} k_{y}^{2}=0$. The solution is $\frac{k_{x}}{k_{y}}=\frac{-\zeta_{x y} \pm \sqrt{\zeta_{x y}^{2}-4 \zeta_{x} \zeta_{y}}}{2 \zeta_{x}}$. If the non-linearity in both planes have the same sign and $4 \zeta_{x} \zeta_{y} \geq \zeta_{x y}^{2}$ we get the absolutely convex (or concave) surface. They both have name of the steep surface. Thus, the maximum stable region is when all $\zeta_{x}, \zeta_{y}, \zeta_{x y}$ have the same sign.

\section{THE LATTICES WITH $\zeta_{X} \gg H_{30 Q}$}

What kind of lattice is needed in order to have a possibility to adjust the detuning $\Delta$, the nonlinear tune shift $\zeta_{x}, \zeta_{x y}, \zeta_{y}$ and the effective sextupole harmonic $h_{k_{x}, k_{y}, q}$ separately. From our point of view the only appropriate lattice is the lattice with the separated functions of the arcs and the straight sections. Such a lattice has some number of superperiods consisting of the arc and the straight sections. Each arc includes eight periodical cells, containing combined function (or usual) bend magnets and focusing (and defocusing) quadrupoles. The tune of the arcs in the horizontal and the vertical planes is integer, $\nu_{x}=3, \nu_{y}=2$.

The sextupoles are placed on arcs periodically . To a first approximation in this condition $h_{k_{x}, k_{y}, q} \rightarrow 0$ (see eq.2), since each $n-t h$ sextupole is compensated by $(n+4)-t h$ in the horizontal and by $(n+2)-t h$ in the vertical planes correspondingly. However, since again the tune is integer, the nonlinear tune shift can be varied in a wide region, since $\left|h_{k_{x}, k_{y}, q}\right|^{2} \rightarrow 0$ and $k_{x} \nu_{x}+k_{y} \nu_{y}-q \rightarrow 0$ simultaneously. The total chromaticity of whole ring is adjusted to zero, but the arc itself has a positive chromaticity, compensated by the negative chromaticity of the straight section. Changing the chromaticity of the arc, we can adjust the required sign and value of the non-linear tune shifts. The working point and the detuning $\Delta$ of whole ring is modified by the tune of the straight section, while the arc remain to be invariable.

Figure 1 shows the dynamic aperture tracking of ASTRID 2[1] versus the tune in the vicinity of the third integer resonance $\nu_{x}=9.33$, when the tune shift $\zeta_{x}=$ 100 , the effective harmonic $h_{30 q}=0.1$ and the detuning equals to $-0.02 ;-0.01$ and 0.01 correspondingly. During small change of the detuning the system runs through three stages, what is exactly in accordance with the Hamiltonian described above. In the early stage the system has four separated islands with four centres and three saddles. In the middle stage all four islands are surrounded by the stable trajectories. After change of the detuning sign the system has one fixed point. In case of a negative tune shift the 

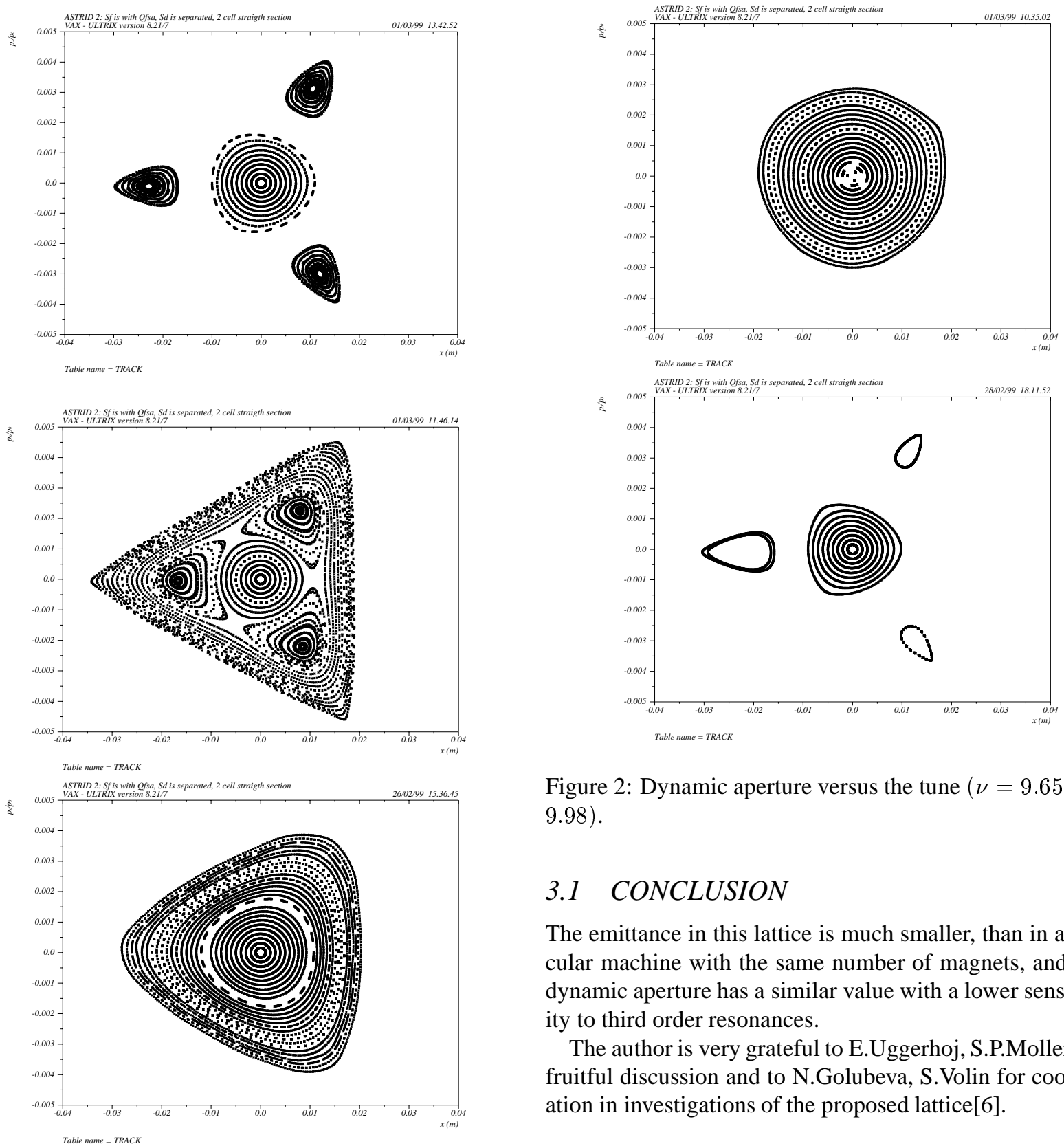

Figure 2: Dynamic aperture versus the tune $(\nu=9.65$ and $9.98)$.

\subsection{CONCLUSION}

The emittance in this lattice is much smaller, than in a circular machine with the same number of magnets, and the dynamic aperture has a similar value with a lower sensitivity to third order resonances.

The author is very grateful to E.Uggerhoj, S.P.Moller for fruitful discussion and to N.Golubeva, S.Volin for cooperation in investigations of the proposed lattice[6].

\section{REFERENCES}

Figure 1: Dynamic aperture versus the detuning $(\Delta=$ $-0.02 ;-0.01 ; 0.01)$.

first and last stages are exchanged with each other. Moving away from the resonance $\nu_{x}=9.33$, the system appears in the vicinity of the next resonance $\nu_{x}=10.0$. Figure 2 (upper picture) shows the dynamic aperture in the middle of way between two resonances, when they compensate each other, and the triangular shape transforms into the oval. Approaching to the resonance $\nu_{x}=10.0$, the dynamic aperture becomes similar to the starting point (lower picture, fig.2). The behavior of the dynamic aperture has the periodic character. The working point is chosen slightly higher or lower than the resonance $\nu_{x}=9.33$ under positive or negative tune shift correspondingly.

[1] Yu. Senichev, The proposed racetrack lattice for the synchrotron light source ASTRID II, EPAC-98, Stockholm.

[2] K.L.Brown, Second-Order Magnetic Optical Achromat, IEEE Trans.Nucl.Sci., NS-26(3), p.3490, 1979.

[3] G.Guignard, J.Hagel, Sextupolar correction and dynamic aperture: numerical and analytical tools, Particle Accelerator, 1986, vol.18, pp.129-165.

[4] S.Volin, Coping with high order perturbation effects in the particle dynamics near 1/3-integer resonance, preprint INR0863/94, 40 pages.

[5] N.Nekhoroshev, Method of successive canonical replacement of variables, UMN 32/6 (1977).

[6] Lattice Study of the ASTRID II Synchrotron Light Source, Internal report of INR, 1999. 